\title{
Prevalence and Clinical Outcomes of Dystrophin associated Dilated Cardiomyopathy without severe skeletal myopathy
}

Short title: Natural history of $D M D$ DCM without severe skeletal myopathy

Maria Alejandra Restrepo-Cordoba, MD ${ }^{1,2,3}$; ale_res@hotmail.com

Karim Wahbi, MD, $\mathrm{PhD}^{4}$; karim.wahbi@aphp.fr

Anca R. Florian, MD5; ancarezeda.florian@ukmuenster.de

Juan Jiménez-Jáimez, MD, PhD6; jimenez.jaimez@gmail.com

Luisa Politano, MD, $\mathrm{PhD}^{7}$; luisa.politano@unicampania.it

Michael Arad, MD ${ }^{8}$; michael.arad@sheba.health.gov.il

Vicente Climent Paya, MD, $\mathrm{PhD}^{9}$; vcliment@coma.es

Ana Garcia-Alvarez, MD, PhD ${ }^{2,10}$; anagarci@clinic.cat

Rasmus Bastkjaer Hansen, MD ${ }^{11}$; rasmus@ bastkjaer.dk

José M. Larrañaga-Moreira, MD ${ }^{12}$; c.larranaga88@gmail.com

Milos Kubanek, MD, PhD ${ }^{13}$; mikb@ikem.cz

Luis Rocha Lopes, MD, PhD ${ }^{3,14,15}$; luisrlopes@hotmail.com

Andrea Ros, $\mathrm{MSc}^{16}$; arosp.germanstrias@gencat.cat

Ruxandra Jurcut, MD, PhD ${ }^{3,17}$; rjurcut@ gmail.com

Torsten B. Rasmussen, MD, $\mathrm{PhD}^{18}$; torasm@rm.dk

Luis Ruiz-Guerrero, MD ${ }^{19}$; luruizguerrero@gmail.com

Regina Pribe-Wolferts, MD ${ }^{20}$; regina.pribe-wolferts@med.uni-heidelberg.de Julian Palomino-Doza, MD, PhD ${ }^{2,21}$; julian.palomino@salud.madrid.org

Zofia Bilinska, MD, PhD²2; z.bilinska@ikard.pl

José F Rodríguez-Palomares, MD, PhD²,23; jfrodriguezpalomares@ gmail.com

Rosa LE Van Loon, MD, $\mathrm{PhD}^{24}$; r.l.e.vanloon@umcutrecht.nl 
María Teresa Basurte Elorz, MD²5; maytebasurte@yahoo.es

Giovanni Quarta, MD, $\mathrm{PhD}^{26}$; giovanni.quarta@yahoo.it

Maria Robledo Iñarritu, MD²7; maria.robledoinarritu@osakidetza.eus

Job A.J. Verdonschot, MD, MSc ${ }^{28}$; job.verdonschot@mumc.nl

Tanya Stojkovic, MD, $\mathrm{PhD}^{29}$; tanya.stojkovic@aphp.fr

Zornitsa Shomanova, MD5; zornitsa.shomanova@ukmuenster.de

Francisco Bermudez-Jimenez, MD, PhD ${ }^{6}$; derbifdx@gmail.com

Alberto Palladino, MD ${ }^{7}$; alberto.palladino@libero.it

Dov Freimark MD ${ }^{8}$; Dov.Freimark@sheba.health.gov.il

Maria I. García-Álvarez, MD9; migarciaalvarez@ hotmail.com

Paloma Jorda, MD ${ }^{10}$; pjordab@ clinic.cat

Fernando Dominguez, MD, PhD ${ }^{1,2,3}$; fdominguezrodriguez@gmail.com

Juan Pablo Ochoa, MD ${ }^{1,30}$; juanpablo.ochoa@healthincode.com

Francesca Girolami, MD ${ }^{31}$; francesca.girolami@meyer.it

Ramon Brugada, MD, $\mathrm{PhD}^{2,32,33}$; rbrugada@idibgi.org

Benjamin Meder, MD, PhD ${ }^{20,34}$; benjamin.meder@med.uni-heidelberg.de

Roberto Barriales-Villa, MD, $\mathrm{PhD}^{2,12}$; rbarrialesv@gmail.com

Jens Mogensen, MD, $\mathrm{PhD}^{11}$; jens.mogensen@rsyd.dk

Pascal Laforêt, MD, PhD ${ }^{35}$; pascal.laforet@aphp.fr

Ali Yilmaz, MD ${ }^{5}$; ali.yilmaz@ukmuenster.de

Perry Elliott, MD, FRCP ${ }^{3,14,15}$; perry.elliott@ucl.ac.uk

Pablo Garcia-Pavia, MD, PhD ${ }^{1,2,3,36,37}$; pablogpavia@yahoo.es

For the European genetic cardiomyopathies initiative investigators (Authors listed in the appendix). 


\section{Departments and Institutions}

1. Heart Failure and Inherited Cardiac Diseases Unit. Department of Cardiology. Hospital Universitario Puerta de Hierro, Madrid, Spain.

2. Centro de Investigación Biomédica en Red en Enfermedades Cardiovasculares (CIBERCV), Madrid, Spain.

3. European Reference Network for Rare and Low Prevalence Complex Diseases of the Heart (ERN-GUARDHEART).

4. APHP, Cochin Hospital, Cardiology Department, FILNEMUS, Paris-Descartes, Sorbonne Paris Cité University, 75006 Paris, France.

5. Division of Cardiovascular Imaging, Department of Cardiology I, University Hospital Muenster, Muenster, Germany.

6. Cardiology Department. Hospital Universitario Virgen de las Nieves. Granada, Spain.

7. Cardiomyology and Medical Genetics - University of Campania Luigi Vanvitelli, Naples, Italy.

8. Leviev Heart Center, Sheba Medical Center and The Sackler Faculty of Medicine, Tel Aviv University, Israel.

9. Cardiology Department, University General Hospital of Alicante, Alicante, Spain.

Institute of Health and Biomedical Research (ISABIAL), Alicante, Spain.

10. Institut Clinic Cardiovascular. IDIBAPS. Hospital Clínic, University of Barcelona, Barcelona, Spain.

11. Department of Cardiology, Odense University Hospital, Denmark.

12. Inherited Cardiovascular Diseases Unit, Cardiology Service, Complexo Hospitalario Universitario de A Coruña, Servizo Galego de Saúde (SERGAS), Instituto de Investigación Biomédica de A Coruña (INIBIC), Universidade da Coruña, A Coruña, Spain.

13. Department of Cardiology, Institute for Clinical and Experimental Medicine, Prague, Czech Republic.

14. Barts Heart Centre, St Bartholomew's Hospital, Barts Health NHS Trust, London, United Kingdom.

15. Centre for Heart Muscle Disease, Institute of Cardiovascular Science, University College London, United Kingdom.

16. Cardiogenetics Unit, Clinical Genetics Department, University Hospital Germans Trias i Pujol, Badalona, Spain. 
17. Expert Center for Rare Cardiovascular Genetic Diseases, 3rd Cardiology Department, Emergency Institute for Cardiovascular Diseases "Prof Dr. C.C. Iliescu", Bucharest, Romania.

18. Department of Cardiology, Aarhus University Hospital, Aarhus, Denmark.

19. Department of Cardiology, Hospital Universitario Marqués de Valdecilla, IDIVAL, Santander, Spain.

20. Institute for Cardiomyopathies Heidelberg, University Hospital Heidelberg, Heidelberg, Germany.

21. Inherited cardiac diseases unit, Cardiology Department, Hospital Universitario 12 de Octubre, Instituto de Investigación i+12. Madrid. Spain.

22. Unit for Screening Studies in Inherited Cardiovascular Diseases. National Institute of Cardiology, Warsaw, Poland.

23. Hospital Universitari Vall d'Hebron, Department of Cardiology. Vall d'Hebron Institut de Recerca (VHIR). Universitat Autònoma de Barcelona, Barcelona, Spain.

24. Department of Genetics, University Medical Center Utrecht, University of Utrecht, The Netherlands.

25. Heart Area. Complejo Hospitalario de Navarra, Pamplona, Spain.

26. Cardiology Department, ASST Papa Giovanni XXIII, Bergamo, Italy.

27. Hospital Universitario Araba - Txagorritxu, Vitoria, Spain.

28. Department of Cardiology and Department of Clinical Genetics, Maastricht University Medical Center, Maastricht, The Netherlands.

29. Referral Center of Neuromuscular Diseases, Myology Institute, Pitié-Salpêtrière Hospital, Paris, France.

30. Cardiology Department, Health in Code, A Coruña, Spain

31. Department of Paediatric Cardiology Meyer Children's Hospital, Florence Italy

32. Inherited Cardiac Diseases Unit. Department of Cardiology. Hospital Universitari Dr Josep Trueta, Girona, Spain.

33. Medical Science Department, School of Medicine, University of Girona, Spain.

34. Stanford University School of Medicine, Department of Genetics, California, USA

35. APHP, Hôpital Raymond Poincaré, Centre de Référence des maladies neuromusculaires Nord-Est-Île de France, Garches, France. 
36. Universidad Francisco de Vitoria (UFV), Pozuelo de Alarcon, Spain.

37. Centro Nacional de Investigaciones Cardiovasculares (CNIC), Madrid, Spain.

Word count: 3762 (includes text, excludes abstract, references, tables and figures).

Funding: This work was supported by grants from the following institutions: Instituto de Salud Carlos III (ISCIII) (PI17/01941, AC16/0014) to PGP. Research grants of the Ministry of Health, Czech Republic [MZ AZV 15-27682A], [NV19-08-00122] and $M H$ CZ - DRO ("Institute for Clinical and Experimental Medicine - IKEM, IN 00023001 ").Grants from the Deutsches Zentrum für Herz-Kreislauf-Forschung (German Center for Cardiovascular Research, DZHK) and Informatics for Life (Klaus Tschira Foundation) to BM. The European Union (DETECTIN-HF and GENPROVIC projects from ERA-CVD framework to $\mathrm{ZB}$ and $\mathrm{BM}$ and PGP, respectively. MAR-C was supported by ISCIII by an IPFIS contract (IFI17/003). LRL is funded by a Medical Research Council Clinical Academic Partnership (CARP) award. BM was supported by an excellence fellowship of the Else Kröner Fresenius Foundation.

Grants from ISCIII and the Spanish Ministry of Economy and Competitiveness are supported by the Plan Estatal de I.D.I 2013-2016 - European Regional Development Fund (FEDER) "A way of making Europe". The Hospital Universitario Puerta de Hierro,

the Emergency Institute for Cardiovascular Diseases "Prof Dr. C.C. Iliescu" and Saint Bartholomews' Hospital are members of the European Reference Network for rare, lowprevalence, and complex diseases of the heart (ERN GUARD-Heart).

Disclosures: none

\section{Correspondence:}

Pablo Garcia-Pavia, MD, PhD

Heart Failure and Inherited Cardiac Diseases Unit,

Department of Cardiology

Hospital Universitario Puerta de Hierro

Manuel de Falla, 2; 28222 Madrid, Spain.

Phone: (+ 34) 911917297 Fax: (+34) 911917718

e-mail: pablogpavia@yahoo.es

twitter: @dr_pavia 


\begin{abstract}
AIMS: Dilated cardiomyopathy (DCM) associated with dystrophin gene (DMD) mutations in individuals with mild or absent skeletal myopathy are often indistinguishable from other DCM forms. We sought to describe the phenotype and prognosis of $D M D$ associated DCM in DMD mutation carriers without severe skeletal myopathy.
\end{abstract}

METHODS AND RESULTS: At 26 European centers, we retrospectively collected clinical characteristics and outcomes of 223 DMD mutation carriers ( $83 \%$ males, $33 \pm 15$ years). 112 individuals (52\%) had DCM at first evaluation $(n=85 ; \mathrm{LVEF}=34 \pm 11.2 \%)$ or developed DCM ( $\mathrm{n}=27$; LVEF 41.3 $\pm 7.5 \%$ ) after a median follow-up of 96 months (IQR: 5-311 months). DCM penetrance was $45 \%$ in carriers older than 40 years. DCM appeared earlier in males and was independent of the type of mutation, presence of skeletal myopathy, or elevated serum creatine kinase levels. Major adverse cardiac events (MACE) occurred in $22 \%$ individuals with DCM, 18\% developed end-stage heart failure and 9\% SCD or equivalent. Skeletal myopathy was not associated with survival free of MACE in patients with DCM. Decreased left ventricle ejection fraction and increased left ventricular end-diastolic diameter at baseline were associated with MACE. Individuals without DCM had favorable prognosis without MACE or death during follow-up.

CONCLUSIONS: $D M D$-associated DCM without severe skeletal myopathy is characterized by incomplete penetrance but high risk of MACE, including progression to end-stage heart failure and ventricular arrhythmias. DCM onset is the major determinant of prognosis with similar survival regardless of the presence of skeletal myopathy.

\title{
Abstract Word count: 235
}

KEY WORDS: Dystrophin, DMD, dilated cardiomyopathy, heart failure, myopathy. 
'One-sentence Summary'

$D M D$-associated DCM without severe skeletal myopathy is characterized by incomplete penetrance but high risk of MACE, including progression to end-stage heart failure and ventricular arrhythmias. 


\section{INTRODUCTION}

Dilated cardiomyopathy (DCM) is characterized by left ventricular (LV) enlargement and systolic dysfunction that cannot be attributed to abnormal loading conditions or coronary artery disease. ${ }^{1}$ Genetic testing can identify a mutation in $40-50 \%$ of DCM patients, affecting $>40$ genes that encode a heterogeneous group of proteins. ${ }^{2}$

Mutations in the $D M D$ gene that codes for the cytoskeletal protein dystrophin cause both DCM and skeletal myopathy (Duchenne and Becker's muscular distrophy) independently or in combination. ${ }^{3,4}$

Individuals with Duchenne muscular dystrophy, the most aggressive form of dystrophinopathy, present with skeletal muscle involvement early in childhood and loss of ambulation by 10 to 12 years of age ${ }^{5}$. Cardiac involvement in this population is well characterized, with end stage heart failure (HF) and respiratory failure as the main causes of death. ${ }^{4,6,7}$ In contrast, there are less data about cardiac disease in patients with milder skeletal myopathy phenotype (Becker muscular dystrophy [BMD]), ${ }^{4,8,9}$ and in those with isolated DCM without skeletal muscle impairment. ${ }^{3}$

Available data on the clinical characteristics and prognosis of cardiac disease in patients with $D M D$ mutations mostly derive from patients evaluated because of skeletal muscle disease but, in the absence of obvious muscular involvement, cardiomyopathy of $D M D$ mutation carriers could be clinically indistinguishable from other types of DCM.

Given the development of specific disease-modifying therapies to correct certain DMD mutations, ${ }^{10-12}$ there is a new imperative to better characterize DMD-associated DCM to facilitate tailored medical therapy.

This study sought to describe the clinical profile and long-term cardiac outcomes of DCM patients and asymptomatic relatives with $D M D$ mutations without severe skeletal 
myopathy by analysing a large cohort of patients recruited from an established international multicenter collaboration. ${ }^{13-15}$

\section{METHODS}

A chart review was performed in all patients (probands and available relatives) with a pathogenic or likely pathogenic $D M D$ mutation followed at 26 referral centers (Supplementary material). Individuals were evaluated after myopathy and/or DCM diagnosis, or as part of family screening. Only individuals $>13$ years without diagnosis of Duchenne muscular dystrophy and who had at least one cardiac evaluation were included. Neuromuscular specialists at each center assessed the patients clinically. The presence of abnormal muscle examination together with loss of independent ambulation by the age of 13 years was consistent with the diagnosis of Duchenne muscular dystrophy $^{4,5}$ (supplementary material).

Data from the first and last clinical contact at each center were recorded. All patients had planned reviews every 6-12 months or more frequently if clinically indicated. The followup for each patient was calculated from the date of first evaluation at a participating center, to the occurrence of a study endpoint, death from another cause, or the date of the most recent evaluation. DCM was defined as a left ventricular ejection fraction (LVEF) $<50 \%{ }^{1}$

Clinical, echocardiographic, electrocardiographic, Holter, and genetic data were collected. When available, data from the first cardiac magnetic resonance (CMR) were also obtained. Details of clinical events occurring before the first clinical contact and during follow-up (including the timing of events) were recorded. Information about heart failure therapy at the last follow-up was collected in patients with DCM. 
Clinical events were defined as follows: device implantation (pacemaker, implantable cardioverter-defibrillator [ICD], or cardiac resynchronization therapy); supraventricular tachycardia (atrial fibrillation $[\mathrm{AF}]$ and atrial flutter); HF admission; left ventricular assist device (LVAD) implantation, heart transplantation (HT), sustained ventricular tachycardia (SVT), appropriate ICD shock, sudden cardiac death (SCD), aborted SCD, and death from any other cardiac or non-cardiac cause.

Major adverse cardiac events (MACE) were defined as a composite of appropriate ICD shock, aborted SCD, SCD, LVAD implantation, HT and cardiac death. A composite of end-stage HF events (cardiac death secondary to HF progression, HT, and LVAD implantation) and of ventricular arrhythmic complications (SCD, aborted SCD and ICD shock) were also constructed.

Presence of muscular weakness and serum creatine kinase (CK) levels were recorded when available, as well as date of BMD diagnosis when performed.

\section{Genetic analysis}

Deoxyribonucleic acid sequence analysis was performed through the participating institutions. Variants were categorized as copy number variations (CNVs) (deletions and duplications) and non-CNVs. Non-CNVs were subdivided in truncating (splicing, nonsense and frameshift variants) and non-truncating (missense and in-frame deletion variants). Pathogenicity of variants was established according to the current American College of Medical Genetics and Genomics guidelines. ${ }^{16,17}$

\section{Statistical analysis}

Continuous variables are presented as mean and standard deviation or median and interquartile ranges (IQR), as appropriate. Categorical data are reported as counts and 
percentages. For statistical analysis, Chi-square test or Fisher exact test were used for categorical variables. Student's t-test and the Mann-Whitney nonparametric test were used in 2-group comparisons. Cumulative event-free survival was evaluated from baseline evaluation with Kaplan-Meier curves and hazard ratios were estimated by Cox proportional hazards regression. Univariate Cox proportional hazards models were created to identify factors associated with MACE during follow-up. Multivariable Cox proportional hazards modeling with variables that were statistically significant at the 0.05 $\alpha$-level was subsequently performed to identify independent factors associated with MACE during follow-up. Survival was calculated from birth. A 2-sided p-value $<0.05$ was considered statistically significant. Analysis was conducted using the 7 Stata SE package (version 14, Stata Corp, College Station, Texas). 


\section{RESULTS}

The study cohort comprised 223 individuals (146 probands and 77 relatives; $83 \%$ males, $33 \pm 15$ years at first evaluation) from 178 families (range 1 to 9 individuals per family) firstly clinically evaluated from 1987 to 2018. Most individuals (172, 77\%) had CNVs (deletions and duplications) in DMD. Non-CNVs were found in 51 patients (23\%), of which $45(20 \%)$ were truncating and $6(3 \%)$ were non-truncating (table $1 \mathrm{~S}$ and table $2 \mathrm{~S})$.

\section{DCM phenotype and changes in LV function during follow-up}

At baseline evaluation, 85 patients (38\%) had DCM (52 with and 33 without skeletal myopathy) and $92(41 \%)$ had isolated skeletal muscle disease. The remaining 46 individuals (21\%) had a normal clinical phenotype. Most individuals were evaluated because of symptoms $(\mathrm{n}=138)$, including 16 heart failure admissions. 43 patients were evaluated in the context of family screening. Individuals with DCM at baseline were diagnosed at a mean age of $34.6 \pm 13.5$ years (range 13 to 77 years). Findings in patients with DCM at first evaluation are shown in table 1. Most (97\%) were in sinus rhythm, $20 \%$ had QRS duration $>120 \mathrm{~ms}$ and 39\% showed negative T waves (mainly in inferior and inferolateral leads). Of note, LV wall motion abnormalities were reported in $46 \%$ of cases and isolated right ventricular involvement in the absence of DCM was not observed. 213 subjects were followed for a median of 96 months (IQR: 5-311 months) and 10 patients were lost to follow-up. A total of 27 individuals who had normal cardiac function at baseline developed DCM (85\% males, mean age at DCM diagnosis $39.8 \pm 15.7$ years), with a time interval between the first evaluation and DCM diagnosis of 7 years (IQR: 214) (Figure 1). The presence of negative $T$ waves on baseline ECG was the only baseline feature associated with DCM onset during follow up (40\% vs 14\%, p=0.003) (table 3S). 
Final occurrence of DCM in the entire cohort was $52 \%(n=112)$, with a mean age of diagnosis of $34.9 \pm 14.1$ years (range 13-77 years). DCM penetrance by 30 and 40 years were $24 \%$ and $45 \%$, respectively. Neither the clinical evidence of skeletal myopathy nor increased CK levels were associated with DCM appearance but DCM prevalence was higher among probands than relatives $(57.5 \%$ vs $36.6 \%, \mathrm{p}<0.01)$ (tables $3 \mathrm{~S}$ and $4 \mathrm{~S})$. Type of mutation was not associated with DCM diagnosis at any point and mean age at DCM diagnosis did not differ between type of mutations $(34.7 \pm 13.9,34.0 \pm 15.0$ and $41.6 \pm 14.0$ years in $\mathrm{CNV}$, truncating and non-truncating variants, respectively; $\mathrm{p}=0.4$ ). Clinical characteristics and adverse events among patients carrying the three genetic variants present in $>10$ individuals were similar and comparable with the rest of the cohort (table $5 \mathrm{~S})$.

Concerning medical treatment of DCM patients, $94(85.4 \%)$ and $13(12.6 \%)$ subjects were treated with ACEI/ARB and sacubitril/valsartan, respectively, 98 (88.2\%) received beta-blockers, 59 (53.6\%) were on mineralocorticoid receptor antagonists (MRAs) and 9 (8\%) were on ivabradine. Only 4 patients were not receiving any HF medication due to hypotension and intolerance.

Forty patients with DCM at initial evaluation presented a severely reduced LVEF at last follow-up (53\% of patients with LVEF at last follow-up available) and only $6(8 \%)$ with DCM at initial evaluation normalized LVEF.

CMR studies were available in 145 patients (59 with DCM and 86 without DCM). CMR findings are shown in Table 6S. A total of $112(85.5 \%)$ exhibited LGE without differences in the presence of LGE between patients with $(91 \%)$ and without DCM $(82 \%)(p=0.2)$. The presence of LGE in individuals without DCM was not associated with subsequent DCM diagnosis during follow-up ( $8 \%$ vs $7 \%$ of patients with new-onset DCM in LGE positive vs LGE negative groups; $\mathrm{p}=1.0$ ). 


\section{Clinical events}

A total of 25 individuals, all with DCM, (22\% of DCM patients, $92 \%$ males) had MACE and 8 died ( $48 \pm 20$ years). The causes of death were progressive HF ( $n=5 ; 62 \%), \operatorname{SCD}$ $(n=1)$, transplant rejection $(n=1)$ and non-cardiac $(n=1)$. Mean age at MACE was $37.4 \pm 14.9$ years (IQR:24-48) and $84 \%$ of MACE occurred in individuals $\leq 50$ years. Figures $2 \mathrm{~A}$ and $2 \mathrm{~B}$ show survival curves for MACE for the entire cohort and for individuals with and without DCM. No adverse events were reported in patients without DCM.

The end-stage HF composite endpoint was reached in 20 individuals (18\% of DCM patients, mean age at event $36.2 \pm 15.2$ years). At the end of follow-up, 34 patients (16\%) had been admitted with HF and $21(10 \%)$ were in NYHA class III-IV with 13 individuals showing worse NYHA class. Fifteen patients received a HT (7\%; mean age $35 \pm 16$ years) and $3(1.5 \%)$ required LVAD implantation ( 2 as a bridge to HT and one as destination therapy). Among the 5 patients who died from end-stage HF one died while on HT waiting list due to donor shortage while the remaining 4 had other comorbidities that precluded inclusion in HT waiting list.

The composite endpoint of arrhythmic events was reached by 10 individuals ( $9 \%$ of DCM patients, mean age at event $40.5 \pm 11.1$ years). SVT occurred in 6 patients without ICD and 1 patient had an aborted SCD on presentation. Of the 112 patients with DCM, 40 (36\%) received an ICD (85\% for primary prevention), and $18(16 \%)$ received a CRT device. Nine patients had at least one appropriate ICD shock ( 8 for SVT and 1 for ventricular fibrillation) and one patient died because of an arrhythmic event. Of note, we did not observe any clinical characteristics significantly different between ICD patients with and without arrhythmic events. 
A total of 20 patients (10\%) developed AF, and 7 received a pacemaker ( 3 for AV block, 2 after AV node ablation, and 2 for unknown cause). Neurologic events were reported in 7 patients with DCM, including 5 transient ischemic attacks and 2 strokes.

\section{DCM with skeletal myopathy vs isolated DCM}

Among 112 individuals who had DCM, 33 showed isolated DCM (67\% males) whereas 79 had DCM in combination with skeletal myopathy (99\% males). Clinical characteristics are shown in Table 2. Patients with isolated DCM were evaluated for the first time later $(41.9 \pm 17.6$ vs $30.5 \pm 11.9$ years; $p=0.001)$ and exhibited an older mean age at DCM diagnosis $(41.6 \pm 18$ years vs $32.1 \pm 11.2$ years; $p=0.01)$. Baseline and final LVEF were similar in both groups while final left ventricular end-diastolic diameter (LVEDD) was greater among those with isolated DCM $(60.5 \pm 9.7$ vs $65.4 \pm 12.5 \mathrm{~mm} ; \mathrm{p}<0.01)$. The distribution of $D M D$ mutations did not differ between groups. Increased CK levels $(>200$ UI/L) were observed only in $48 \%$ and $71 \%$ of patients with isolated DCM at baseline and last follow-up, respectively.

Although MACE were more frequent in patients with isolated DCM than in those with concomitant skeletal myopathy $(35.5 \%$ vs $17.7 \%$; $p=0.046)$, there was no difference in survival free from MACE between both groups (Figure 2C). A detailed list of events can be found in Table 7S.

\section{DCM phenotype and clinical events in females}

A total of 37 female carriers were included in the study (9 index patients; $62 \%$ carrying CNVs; $44 \pm 16$ years at first evaluation). In 8 women DCM was present at initial evaluation and 4 developed DCM during follow-up (DCM occurrence 32\%). Only one female patient with DCM had concomitant skeletal myopathy (1/12, 8\%) (online figure 1). Age 
at diagnosis of DCM was $52.2 \pm 16.9$ years, later than in men $(32.8 \pm 12.3 ; \mathrm{p}<0.001)$. Distribution of age at DCM diagnosis by sex is shown in Figure 3. In 7 females with $\operatorname{DCM}(58 \%)$ cardiac symptoms were the reason for initial evaluation and were the index cases in their families ( $52 \pm 18$ years at diagnosis). Only 3 women had BMD (in one case associated with DCM and preceding the diagnosis). Concerning clinical events, four patients had AF at baseline and 2 received a pacemaker during follow-up ( 1 for AV block and 1 for unknown cause) and one had a transient ischemic attack (in absence of AF). All events occurred in patients with DCM. Lastly, 2 women with DCM and without skeletal involvement had MACE (1 died due to end-stage HF and 1 received an appropriate ICD shock).

\section{Predictors of adverse events}

DCM patients with MACE and the end-stage HF composite endpoint tended to be younger at DCM diagnosis and had lower LVEF and higher LVEDD at first evaluation than DCM patients without MACE (Table 3; Table 8S). In contrast, patients with arrhythmic complications had similar age at DCM diagnosis, but still had lower LVEF and larger LVEDD at baseline compared with DCM patients without arrhythmic events (Table 9S). ECG features, CK levels and the presence of LGE did not differ between patients with or without MACE (Table 3), end-stage HF or ventricular arrhythmic events (Tables 8S and 9S).

Finally, LVEF at baseline and age at DCM diagnosis were the only factors associated with MACE identified at multivariate Cox analysis (Table 10S). 


\section{DISCUSSION}

In this large multicenter study of individuals with $D M D$ mutations and without severe skeletal myopathy, $52 \%$ of patients had DCM at baseline or developed DCM during a median follow-up of 96 months, with an aggressive clinical course characterized by endstage HF and a non-negligible arrhythmic risk. The presence of DCM was the major determinant of prognosis and survival free of MACE was similar in DCM patients with and without skeletal myopathy (Graphical Abstract). ECG, echocardiographic features, LGE, CK levels and mutation type did not identify individuals who developed DCM during follow up.

Dystrophin is a cytoplasmic structural protein essential to stabilize the interaction between the cytoskeleton, the cell membrane and the extracellular matrix. ${ }^{4} \mathrm{~A}$ lack of dystrophin results in sarcolemmal instability during the repeated cycles of contraction and relaxation with increased susceptibility to injury and fiber necrosis. Cardiac disease has been described in up to $70 \%$ of patients with BMD with a prevalence of DCM ranging between $21 \%$ and $66 \% .^{4,6,9} D M D$-associated DCM with mild or absent skeletal involvement are often indistinguishable from other forms of DCM so the real incidence is unknown. Moreover, in the absence of overt muscular phenotype or in the case that cardiomyopathy precedes muscle involvement, these patients are often evaluated in nonspecialized cardiology clinics with little information about natural history and outcomes. Our study is the first to provide data on age-related penetrance of DCM in these patients. When compared to other genetic forms of DCM, age-related penetrance of DCM in DMD mutations carriers is lower compared to $F L N C, B A G 3$ and $L M N A$ associated DCM in which penetrance ranges between $60 \%$ to $100 \% .{ }^{14,18,19}$ However, the mean age of DCM diagnosis is 35 years, which is comparable to other forms of inherited DCM but earlier than in DCM caused by TTN truncating variants (TTNtv). ${ }^{13}$ 
The use of standard medical HF therapy is currently indicated as soon as DCM is demonstrated in DMD mutations carriers, and early initiation of ACEI before LV dysfunction appearance has shown to prevent ventricular remodeling and decrease mortality in Duchenne patients ${ }^{4,11}$. Although most patients with DCM in our cohort were treated with standard HF therapies, only $8 \%$ patients normalized LVEF and more than half progressed to severely reduced LVEF on follow-up in spite of active management. In line with this low rate of reverse remodeling, this study also shows that the prognosis is poor, with more than $20 \%$ of DCM patients suffering MACE, most of which were endstage $\mathrm{HF}$ events. These results suggest that $D M D$-associated DCM is a more aggressive disease than that caused by, for example, TTNtv, but similar to that described in LMNA and $B A G 3$ associated DCM, despite differences in cohort compositions limit comparisons across published cohorts. ${ }^{13,14,18}$ Further studies would be needed to definitively assess what is the response to guideline-directed heart failure therapies by each genotype.

Although the incidence of clinical relevant arrhythmias seems to be lower than that observed in $L M N A, R B M 20$, and FLNC associated DCM, ${ }^{2,18,19}$ the incidence of ventricular arrhythmias was still relatively high. Of note, no arrhythmic events were reported in individuals with normal LVEF.

The incidence of AF in this young population was also relatively high. The importance of this with respect to anticoagulation is uncertain as only 1 of the 7 individuals with neurologic events had atrial arrythmias. Nevertheless, it seems reasonable to assume that the threshold for considering prophylactic anticoagulants should be low in this population.

We were unable to identify predictors of DCM onset during follow-up except increased number of negative T waves on ECG at baseline evaluation, most on lateral and inferior leads. Typical ECG changes of tall R waves with an increased R/S ratio in lead V1 and 
deep Q waves in leads I, aVL, and V5-6 is well known in dystrophinopathy, but no correlation with DCM presence has been established. ${ }^{4}$ The presence of T wave changes has been described as a nonspecific myocardial damage marker in a BMD $\operatorname{cohort}^{8}$ and abnormal LV repolarization has been reported in X-linked DCM patients, ${ }^{3}$ but this is the first time that ECG findings are reported to be useful in identifying individuals at higher risk of developing DCM and who might benefit from early treatment.

Interestingly, LGE was observed at CMR in a large proportion of patients, but its presence was not associated with subsequent DCM onset or MACE. Transmural pattern of LGE has been previously reported to be associated with adverse events independently of LVEF in muscular dystrophy patients ${ }^{20}$ but LGE pattern and extent were not collected in our study. Recent studies have examined the value of the presence of LGE in predicting outcome in large non-ischemic DCM cohorts (with LVEF $<50 \%$ ), demonstrating that the extent, location and pattern of LGE is strongly associated with an increase in all-cause and cardiac death, mainly SCD and arrhythmic events, even regardless of LVEF values $^{21,22}$. However, these cohorts include patients who may have different genetic, inflammatory or infiltrative substrates, with a well-known heterogeneous behavior and aggressiveness $^{23}$. Additional studies are needed to combine CMR findings with specific genetic substrates for an accurate prognostic stratification of genetic DCM subtypes.

Previous studies in BMD patients showed that the severity and age of onset of cardiomyopathy do not correlate with the severity of musculoskeletal involvement. ${ }^{8}$ Here, we show that there is no difference in cardiac phenotype and survival free of MACE between patients with isolated DCM and those with DCM and concomitant muscular disease. Age at DCM diagnosis and sex distribution were the only clinical variables that 
differed between DCM patients with and without muscular involvement. The older age at DCM diagnosis in patients with isolated DCM could be explained by earlier and more frequent cardiac evaluations of patients with skeletal myopathy.

Regarding gender, $32 \%$ of women with $D M D$ mutations showed DCM at the end of follow-up, two of whom presented MACE. This is comparable to the prevalence reported in series of female carriers evaluated after the diagnosis of a first-degree male relative. ${ }^{11,24,25}$ As observed in other cohorts, female carriers exhibit a variable clinical course ranging from an asymptomatic course to overt DCM with mild symptoms or with end-stage HF or ventricular arrhythmic events. Unlike previous series,${ }^{24-26}$ only 7 female carriers $(19 \%)$ presented with clinically skeletal myopathy and/or elevated CK serum levels and 7 of the 12 female patients with DCM in this cohort were index patients. These findings could explain the older age of DCM diagnosis compared to males, since many women of this cohort were evaluated for the first time for isolated cardiac symptoms. Our results reinforce the need to screen for cardiomyopathy all female $D M D$ mutation carriers, even in absence of symptoms or abnormal CK serum levels and underscore the variable phenotype that can be found in women with $D M D$ mutations ${ }^{25}$.

Currently, $D M D$-associated DCM is recommended to be suspected in the presence of male probands, X-linked recessive inheritance, and increased $\mathrm{CK} .{ }^{2,3}$ In contrast to other reports, ${ }^{3}$ only $48 \%$ of our patients with isolated DCM had elevated CK levels at baseline, and a limited number referred muscular weakness. Furthermore, women accounted for one third of patients with isolated DCM and several women were the first individuals diagnosed in their families. Together our results underscore that clinicians should be aware of the broader and heterogenous clinical spectrum of $D M D$-associated DCM and $D M D$ mutations (including CNVs) should be excluded in DCM patients even in the 
absence of the abovementioned classical features.

\section{Clinical Implications}

This study shows that $D M D$ mutation carriers should be offered life surveillance to diagnose and manage cardiac complications. Patients with $D M D$-associated DCM should receive standard HF therapy, but the poor response seen means that many patients will need prompt assessment for HT or LVAD. ${ }^{27}$

Substantial advances in treatment with various gene therapy approaches have occurred in recent years to improve dystrophin expression in skeletal muscle of DMD patients. ${ }^{10-12}$ However, their impact on cardiac muscle has not been elucidated. The results of the current study show that there is a need to develop efficient therapies to patients with $D M D$ associated DCM and provide a firm basis for clinical trials testing the efficacy of gene therapy in this condition.

\section{Limitations}

Participant centers are all specialized cardiac centers and the study is subject to selection and referral bias. Our study was not designed to evaluate response to individual HF treatments in patients with DCM and preventive treatments in carriers without DCM. Although HF drugs in DCM patients at last follow-up were obtained, time on each drug and mean doses received were not available. Furthermore, we did not collect standardized data on the pattern, distribution, and extent of LGE at CMR and could not evaluate the presence of LV hypertrophy in $D M D$ associated DCM. 


\section{Conclusions}

Penetrance of DCM in DMD mutations carriers without severe skeletal myopathy is incomplete, and expressivity variable. However, developing DCM is the major determinant of prognosis, and is associated with high risk of MACE, including progression to end-stage $\mathrm{HF}$ and ventricular arrhythmias, irrespective of the presence of skeletal myopathy. 


\section{REFERENCES}

1. Pinto YM, Elliott PM, Arbustini E, Adler Y, Anastasakis A, Böhm M, Duboc D, Gimeno J, Groote P de, Imazio M, Heymans S, Klingel K, Komajda M, Limongelli G, Linhart A, Mogensen J, Moon J, Pieper PG, Seferovic PM, Schueler S, Zamorano JL, Caforio ALP, Charron P. Proposal for a revised definition of dilated cardiomyopathy, hypokinetic non-dilated cardiomyopathy, and its implications for clinical practice: a position statement of the ESC working group on myocardial and pericardial diseases. Eur Heart J 2016;37:1850-1858.

2. Hershberger RE, Hedges DJ, Morales A. Dilated cardiomyopathy: the complexity of a diverse genetic architecture. Nat Rev Cardiol 2013;10:531-547.

3. Diegoli M, Grasso M, Favalli V, Serio A, Gambarin FI, Klersy C, Pasotti M, Agozzino E, Scelsi L, Ferlini A, Febo O, Piccolo G, Tavazzi L, Narula J, Arbustini E. Diagnostic Work-Up and Risk Stratification in X-Linked Dilated Cardiomyopathies Caused by Dystrophin Defects. J Am Coll Cardiol 2011;58:925-934.

4. Kamdar F, Garry DJ. Dystrophin-Deficient Cardiomyopathy. J Am Coll Cardiol 2016;67:2533-2546.

5. Bushby K, Finkel R, Birnkrant DJ, Case LE, Clemens PR, Cripe L, Kaul A, Kinnett K, McDonald C, Pandya S, Poysky J, Shapiro F, Tomezsko J, Constantin C. Diagnosis and management of Duchenne muscular dystrophy, part 2: implementation of multidisciplinary care. Lancet Neurol 2010;9:177-189.

6. Tandon A, Jefferies JL, Villa CR, Hor KN, Wong BL, Ware SM, Gao Z, Towbin JA, Mazur W, Fleck RJ, Sticka JJ, Benson DW, Taylor MD. Dystrophin genotype-cardiac phenotype correlations in Duchenne and Becker muscular dystrophies using cardiac magnetic resonance imaging. Am J Cardiol 2015;115:967-971.

7. D’Amario D, Amodeo A, Adorisio R, Tiziano FD, Leone AM, Perri G, Bruno P, 
Massetti M, Ferlini A, Pane M, Niccoli G, Porto I, D’Angelo GA, Borovac JA, Mercuri

E, Crea F. A current approach to heart failure in Duchenne muscular dystrophy. Heart 2017;103:1770-1779.

8. Nigro G, Comi LI, Politano L, Limongelli FM, Nigro V, De Rimini ML, Giugliano MAM, Petretta VR, Passamano L, Restucci B, Fattore L, Tebloev K, Comi L, De Luca F, Raia P, Esposito MG. Evaluation of the cardiomyopathy in becker muscular dystrophy. Muscle Nerve 1995;18:283-291.

9. Jefferies JL, Eidem BW, Belmont JW, Craigen WJ, Ware SM, Fernbach SD, Neish SR, Smith EO, Towbin JA. Genetic Predictors and Remodeling of Dilated Cardiomyopathy in Muscular Dystrophy. Circulation 2005;112:2799-2804.

10. Frank DE, Schnell FJ, Akana C, El-Husayni SH, Desjardins CA, Morgan J, Charleston JS, Sardone V, Domingos J, Dickson G, Straub V, Guglieri M, Mercuri E, Servais L, Muntoni F. Increased dystrophin production with golodirsen in patients with Duchenne muscular dystrophy. Neurology 2020 May 26;94:e2270-e2282.

11. D’Amario D, Gowran A, Canonico F, Castiglioni E, Rovina D, Santoro R, Spinelli P, Adorisio R, Amodeo A, Perrucci G, Borovac J, Pompilio G, Crea F. Dystrophin Cardiomyopathies: Clinical Management, Molecular Pathogenesis and Evolution towards Precision Medicine. J Clin Med 2018;7:291.

12. Garry DJ, Alexy T. Emerging Therapies for Dystrophic Cardiomyopathy. JACC Basic Transl Sci 2019;4:792-794.

13. Akhtar MM, Lorenzini M, Cicerchia M, Ochoa JP, Hey TM, Sabater Molina M, Restrepo-Cordoba MA, Dal Ferro M, Stolfo D, Johnson R, Larrañaga-Moreira JM, Robles-Mezcua A, Rodriguez-Palomares JF, Casas G, Peña-Peña ML, Lopes LR, Gallego-Delgado M, Franaszczyk M, Laucey G, Rangel-Sousa D, Basurte M, PalominoDoza J, Villacorta E, Bilinska Z, Limeres Freire J, Garcia Pinilla JM, Barriales-Villa R, 
Fatkin D, Sinagra G, Garcia-Pavia P, Gimeno JR, Mogensen J, Monserrat L, Elliott PM. Clinical Phenotypes and Prognosis of Dilated Cardiomyopathy Caused by Truncating Variants in the TTN Gene. Circ Heart Fail 2020 Oct;13:e006832.

14. Domínguez F, Cuenca S, Bilińska Z, Toro R, Villard E, Barriales-Villa R, Ochoa JP, Asselbergs F, Sammani A, Franaszczyk M, Akhtar M, Coronado-Albi MJ, Rangel-Sousa D, Rodriguez-Palomares JF, Jiménez-Jáimez J, Garcia-Pinilla JM, Ripoll-Vera T, Mogollón-Jiménez MV, Fontalba-Romero A, Garcia-Medina D, Palomino-Doza J, Gonzalo-Calvo D de, Cicerchia M, Salazar-Mendiguchia J, Salas C, Pankuweit S, Hey TM, Mogensen J, Barton PJ, Charron P, et al. Dilated Cardiomyopathy Due to BLC2Associated Athanogene 3 (BAG3) Mutations. J Am Coll Cardiol 2018;72:2471-2481.

15. Lopez-Sainz A, Dominguez F, Lopes LR, Ochoa JP, Barriales-Villa R, Climent V, Linschoten M, Tiron C, Chiriatti C, Marques N, Rasmussen TB, Espinosa MÁ, Beinart R, Quarta G, Cesar S, Field E, Garcia-Pinilla JM, Bilinska Z, Muir AR, Roberts AM, Santas E, Zorio E, Peña-Peña ML, Navarro M, Fernandez A, Palomino-Doza J, Azevedo O, Lorenzini M, García-Álvarez MI, Bento D, Jensen MK, Méndez I, Pezzoli L, Sarquella-Brugada G, Campuzano O, Gonzalez-Lopez E, Mogensen J, Kaski JP, Arad M, Brugada R, Asselbergs FW, Monserrat L, Olivotto I, Elliott PM, Garcia-Pavia P; European Genetic Cardiomyopathies Initiative Investigators. Clinical Features and Natural History of PRKAG2 Variant Cardiac Glycogenosis. J Am Coll Cardiol. 2020 Jul 14;76:186-197.

16. Richards S, Aziz N, Bale S, Bick D, Das S, Gastier-Foster J, Grody WW, Hegde M, Lyon E, Spector E, Voelkerding K, Rehm HL; ACMG Laboratory Quality Assurance Committee. Standards and guidelines for the interpretation of sequence variants: a joint consensus recommendation of the American College of Medical Genetics and Genomics and the Association for Molecular Pathology. Genet Med 2015 May;17:405-24. 
17. Brandt T, Sack LM, Arjona D, Tan D, Mei H, Cui H, Gao H, Bean LJH, Ankala A, Del Gaudio D, Knight Johnson A, Vincent LM, Reavey C, Lai A, Richard G, Meck JM. Adapting ACMG/AMP sequence variant classification guidelines for single-gene copy number variants. Genet Med 2020 Feb;22:336-344.

18. Kumar S, Baldinger SH, Gandjbakhch E, Maury P, Sellal J-M, Androulakis AFA, Waintraub X, Charron P, Rollin A, Richard P, Stevenson WG, Macintyre CJ, Ho CY, Thompson T, Vohra JK, Kalman JM, Zeppenfeld K, Sacher F, Tedrow UB, Lakdawala NK. Long-Term Arrhythmic and Nonarrhythmic Outcomes of Lamin A/C Mutation Carriers. J Am Coll Cardiol. 2016 Nov 29;68:2299-2307.

19. Ortiz-Genga MF, Cuenca S, Dal Ferro M, Zorio E, Salgado-Aranda R, Climent V, Padrón-Barthe L, Duro-Aguado I, Jiménez-Jáimez J, Hidalgo-Olivares VM, GarcíaCampo E, Lanzillo C, Suárez-Mier MP, Yonath H, Marcos-Alonso S, Ochoa JP, Santomé JL, García-Giustiniani D, Rodríguez-Garrido JL, Domínguez F, Merlo M, Palomino J, Peña ML, Trujillo JP, Martín-Vila A, Stolfo D, Molina P, Lara-Pezzi E, Calvo-Iglesias FE, Nof E, Calò L, Barriales-Villa R, Gimeno-Blanes JR, Arad M, García-Pavía P, Monserrat L. Truncating FLNC Mutations Are Associated With High-Risk Dilated and Arrhythmogenic Cardiomyopathies. J Am Coll Cardiol. 2016 Dec 6;68:2440-2451.

20. Florian A, Ludwig A, Engelen M, Waltenberger J, Rösch S, Sechtem U, Yilmaz A. Left ventricular systolic function and the pattern of late-gadolinium-enhancement independently and additively predict adverse cardiac events in muscular dystrophy patients. J Cardiovasc Magn Reson 2014;16:81.

21. Klem I, Klein M, Khan M, Yang EY, Nabi F, Ivanov A, Bhatti L, Hayes B, Graviss EA, Nguyen DT, Judd RM, Kim RJ, Heitner JF, Shah DJ. The Relationship of LVEF and Myocardial Scar to Long-Term Mortality Risk and Mode of Death in Patients with Non- 
Ischemic Cardiomyopathy. Circulation. 2021 Apr 6;143:1343-1358.

22. Halliday BP, Cleland JGF, Goldberger JJ, Prasad SK. Personalizing Risk Stratification for Sudden Death in Dilated Cardiomyopathy: The Past, Present, and Future. Circulation 2017;136:215-231.

23. Gigli M, Merlo M, Graw SL, Barbati G, Rowland TJ, Slavov DB, Stolfo D, Haywood ME, Dal Ferro M, Altinier A, Ramani F, Brun F, Cocciolo A, Puggia I, Morea G, McKenna WJ, La Rosa FG, Taylor MRG, Sinagra G, Mestroni L. Genetic Risk of Arrhythmic Phenotypes in Patients With Dilated Cardiomyopathy. J Am Coll Cardiol 2019;74:1480-1490.

24. Adachi K, Hashiguchi S, Saito M, Kashiwagi S, Miyazaki T, Kawai H, Yamada H, Iwase T, Akaike M, Takao S, Kobayashi M, Ishizaki M, Matsumura T, Mori-Yoshimura M, Kimura E. Detection and management of cardiomyopathy in female dystrophinopathy carriers. J Neurol Sci 2018;386:74-80.

25. Ishizaki M, Kobayashi M, Adachi K, Matsumura T, Kimura E. Female dystrophinopathy: Review of current literature. Neuromuscul Disord 2018;28:572-581. 26. Florian A, Rösch S, Bietenbeck M, Engelen M, Stypmann J, Waltenberger J, Sechtem U, Yilmaz A. Cardiac involvement in female Duchenne and Becker muscular dystrophy carriers in comparison to their first-degree male relatives: a comparative cardiovascular magnetic resonance study. Eur Heart J - Cardiovasc Imaging 2016;17:326-333.

27. Papa AA, D’Ambrosio P, Petillo R, Palladino A, Politano L. Heart transplantation in patients with dystrophinopathic cardiomyopathy: Review of the literature and personal series. Intractable Rare Dis Res 2017;6:95-101. 


\section{Figures}

Figure 1. Flowchart of the study. Phenotype of individuals with $D M D$ mutations during the study. DCM: dilated cardiomyopathy.

Figure 2. Survival free of MACE. A. Freedom from MACE in all patients included in the study. B. According to the presence of DCM. C. According to muscular phenotype.

Figure 3. Age at DCM diagnosis in individuals with $D M D$ mutations according to gender. Box-plot diagram of age of DCM diagnosis reveals that DCM diagnosis in men is significantly earlier than in women $(\mathrm{p}<0.001)$.

Graphical Abstract. Main Characteristics of DMD-associated DCM. $D M D$-associated DCM shows incomplete penetrance and a later diagnosis among DCM patients without concomitant skeletal myopathy. $D M D$ mutations result in progressive cardiomyocyte death and fibrosis, leading to DCM. DCM onset is the major determinant of prognosis. Prognosis is similar among DMD DCM patients with or without skeletal muscle involvement. 
Table 1. Baseline Characteristics of Individuals with $D M D$ mutations.

\begin{tabular}{|c|c|c|c|c|}
\hline & \multicolumn{4}{|c|}{ DCM at baseline } \\
\hline & $\begin{array}{l}\text { Total Cohort } \\
\qquad(\mathrm{n}=\mathbf{2 2 3})\end{array}$ & $\begin{array}{l}\text { Absent } \\
(n=138)\end{array}$ & $\begin{array}{l}\text { Present } \\
(n=85)\end{array}$ & $p$ value \\
\hline Male & $186(83.4)$ & $109(78)$ & $77(90.5)$ & 0.02 \\
\hline Age at first evaluation, years & $33.3 \pm 15$ & $32.5 \pm 16$ & $34.5 \pm 13.3$ & 0.3 \\
\hline Type of mutation & & & & 0.05 \\
\hline CNVs & 172 & $111(80.4)$ & $61(71.6)$ & \\
\hline Truncating & 45 & $26(18.8)$ & $19(22.3)$ & \\
\hline Non-truncating & 6 & $1(0.7)$ & $5(5.8)$ & \\
\hline CK levels, UI/1 & $1886 \pm 2716$ & $1958 \pm 2450$ & $1754 \pm 3125$ & 0.2 \\
\hline Skeletal Myopathy & $144(64.5)$ & $92(66.6)$ & $52(61.1)$ & 0.4 \\
\hline NYHA III-IV & $18(8.2)$ & $3(2.4)$ & $15(18)$ & $<0.01$ \\
\hline Sustained VT & $6(3.1)$ & 0 & $6(8.7)$ & $<0.01$ \\
\hline ASCD presentation & $2(0.9)$ & 0 & $2(2.3)$ & 0.14 \\
\hline \multicolumn{5}{|l|}{ ECG } \\
\hline Sinus rhythm & $213 / 221(96.3)$ & $133(97)$ & $80(97.5)$ & 1 \\
\hline PR duration, ms & $142.3 \pm 21.1$ & $141.1 \pm 18.5$ & $144.6 \pm 25.5$ & 0.7 \\
\hline QRS duration $>120 \mathrm{~ms}$ & $16(8)$ & $4(3.6)$ & $12(20.3)$ & $<0.01$ \\
\hline Tall $\mathrm{R}$ wave in precordial & $100 / 198(50.5)$ & $73(58.8)$ & $27(36.4)$ & $<0.01$ \\
\hline Pathologic Q waves & $63 / 204(30.8)$ & $43(21)$ & $17(8.3)$ & 0.1 \\
\hline Neg. T-wave any lead & $54 / 206(26.2)$ & $25(19)$ & 29 (39) & $<0.01$ \\
\hline Low QRS in limbs & $17 / 179(9.5)$ & $4(3.3)$ & $13(21.6)$ & $<0.01$ \\
\hline
\end{tabular}

Echocardiogram 
LVEF, \%

$48.9 \pm 15.4$

$59.7 \pm 6.3$

$34 \pm 11.2$

NA

LVEDD, mm

$53.9 \pm 10.1$

$48.3 \pm 6.5$

$62.6 \pm 8.6$

NA

LVWMA

40/203 (19.7)

$6(4.6)$

34 (45.9)

NA

TAPSE, mm

$21.4 \pm 3.7$

$22.5 \pm 3.6$

$20.3 \pm 3.5$

NA

Values are $\mathrm{n}(\%)$ or mean $\pm \mathrm{SD}$. $\mathrm{ASCD}=$ aborted sudden cardiac death; $\mathrm{CNV}=$ copy number variations; $\mathrm{CK}=$ serum creatine kinase; $\mathrm{DCM}=$ dilated cardiomyopathy $\mathrm{ECG}=$ electrocardiogram; LVEDD = left ventricular end-diastolic diameter; LVEF $=$ left ventricular ejection fraction; LVWMA = left ventricle wall motion abnormalities; MR = mitral regurgitation; NA $=$ Not applicable; NYHA $=$ New York Heart Association; TAPSE $=$ tricuspid annular plane systolic excursion; $\mathrm{VT}=$ ventricular tachycardia. 
Table 2. Characteristics of DCM subjects with $D M D$ mutations according to the presence of skeletal myopathy.

DCM with skeletal

myopathy $(n=79)$
Isolated DCM

$\left(n=33^{*}\right)$

p-value

Male

Age at first evaluation, yrs

Age at DCM diagnosis, yrs

Age at last evaluation, yrs

Type of mutation

CNV

Truncating

Non-truncating

CK levels $>200 \mathrm{UI} / \mathrm{L}$ at first evaluation

CK value $>200 \mathrm{UI} / \mathrm{L}$ at last follow up

NYHA III-IV at first evaluation

NYHA III-IV at last follow up

Muscle weakness at first evaluation

Muscle weakness at last follow up

ECG

Sinus rhythm at first evaluation

Sinus rhythm at last follow up

PR interval first evaluation, $\mathrm{mm}$

PR interval follow up, mm

QRS interval first evaluation, $\mathrm{mm}$

QRS interval follow up, mm
$78(98.7)$

$22(66.6)$

$<0.01$

$30.5 \pm 11.9$

$41.9 \pm 17.6$

$<0.01$

$32.1 \pm 11.2$

$41.6 \pm 18$

$<0.01$

$39.8 \pm 12.7$

$47.4 \pm 17.2$

0.01

0.1

60 (75.9)

$22(66.6)$

$14(17.7)$

$11(33.3)$

$5(6.3)$

0

74 (94.8)

$12(48)$

$<0.01$

$69(95.8)$

15 (71.4)

$<0.01$

$9(11.8)$

$8(25.8)$

0.07

$11(14.8)$

9 (29)

0.1

$68(86)$

0

$<0.01$

$72(94.7)$

$2(6.4)$

$<0.01$

$76(100)$

27 (90)

0.02

68 (94.4)

28 (93.9)

1

$140.5 \pm 24.1$

$146 \pm 23$

0.2

$149.2 \pm 22.9$

$162.2 \pm 27.1$

0.05

$102.9 \pm 27.5$

$111.7 \pm 66.4$

0.6

$118.6 \pm 28.5$

$115.3 \pm 35$

0.01 
Tall $\mathrm{R}$ wave precordial first

evaluation

Tall R wave precordial follow up

Negative T-wave any lead first

evaluation

Negative T-wave any lead follow up

Echocardiogram

LVEF at first evaluation, $\%$

LVEF at last follow up, $\%$

LVEDD at first evaluation, $\mathrm{mm}$

LVEDD at last follow up, mm

TAPSE baseline, $\mathrm{mm}$

TAPSE at last follow up, mm
$36(51.4)$

$5(17.8)$

$<\mathbf{0 . 0 1}$

$30(47.6)$

$5(18.5)$

0.01

$29(40.8)$

$29(44.6)$

$10(35.7)$

0.8

$9(34.6)$

0.4

$39.6 \pm 14.4$

$37.5 \pm 10.7$

$59.3 \pm 9.4$

$60.5 \pm 9.7$

$20.7 \pm 3.1$

$20 \pm 4.9$
$62 \pm 9.9$

0.3

$36.6 \pm 13.9$

0.2

0.1

$33.4 \pm 13.8$

$65.4 \pm 12.5$

$<0.01$

$20.4 \pm 4.1$

0.6

$18.3 \pm 4$

0.12

* Data reported at last follow up correspond to 31 patients (2 patients were lost for followup). Abbreviations as in Table 1. 
Table 3. Characteristics of DCM patients with $D M D$ mutations with and without MACE during follow up.

\begin{tabular}{|c|c|c|c|}
\hline & $\begin{array}{l}\text { DCM patients } \\
\text { without MACE } \\
\qquad(n=85)\end{array}$ & $\begin{array}{l}\text { DCM patients } \\
\text { with MACE } \\
(n=25)\end{array}$ & p-value \\
\hline Male & $77(90.5)$ & $23(92)$ & 1 \\
\hline Proband & $61(71.7)$ & $22(88)$ & 0.1 \\
\hline Age at DCM diagnosis, yrs & $35.7 \pm 13.7$ & $30.7 \pm 13.0$ & 0.09 \\
\hline Skeletal Myopathy & $65(76.4)$ & $14(56)$ & 0.04 \\
\hline Isolated DCM & $20(23.5)$ & $11(44)$ & 0.04 \\
\hline \multicolumn{4}{|l|}{ Type of mutation } \\
\hline $\mathrm{CNV}$ & $66(77.6)$ & $14(56)$ & 0.07 \\
\hline Truncating PM & $16(18.8)$ & $9(36)$ & 0.07 \\
\hline Non-truncating PM & $3(3.5)$ & $2(8)$ & 0.07 \\
\hline CK levels al baseline, UI/1 & $2233 \pm 3497$ & $2266 \pm 3689$ & 0.7 \\
\hline CK levels $\geq 200 \mathrm{UI} / \mathrm{L}$ at baseline & $69(84.1)$ & $17(85)$ & 1 \\
\hline NSVT on ECG holter & $17(47)$ & $5(83.3)$ & 0.18 \\
\hline \multicolumn{4}{|l|}{ ECG } \\
\hline QRS duration at baseline, ms & $101.2 \pm 27.8$ & $126.1 \pm 83$ & 0.2 \\
\hline $\begin{array}{l}\text { Tall } \mathrm{R} \text { wave in precordial } \\
\text { leads at baseline }\end{array}$ & $36(45)$ & $5(31.2)$ & 0.4 \\
\hline $\begin{array}{l}\text { Neg T-wave any leads at } \\
\text { baseline }\end{array}$ & $31(38.7)$ & $8(47)$ & 0.5 \\
\hline
\end{tabular}


LVEF at baseline, $\%$

LVEDD at baseline, $\mathrm{mm}$

TAPSE at baseline, $\mathrm{mm}$

Presence of LGE, n (\%)
$42.2 \pm 13.6$

$57.2 \pm 8.2$

$21.3 \pm 3.3$

$52(89.6)$
$27 \pm 10.1$

$<0.01$

$18 \pm 3$

$<0.01$

$3(100)$

Abbreviations as in Table 1 and 2. 
Figure 1. Flowchart of the study. Clinical events and phenotype of individuals during the study. DCM: dilated cardiomyopathy

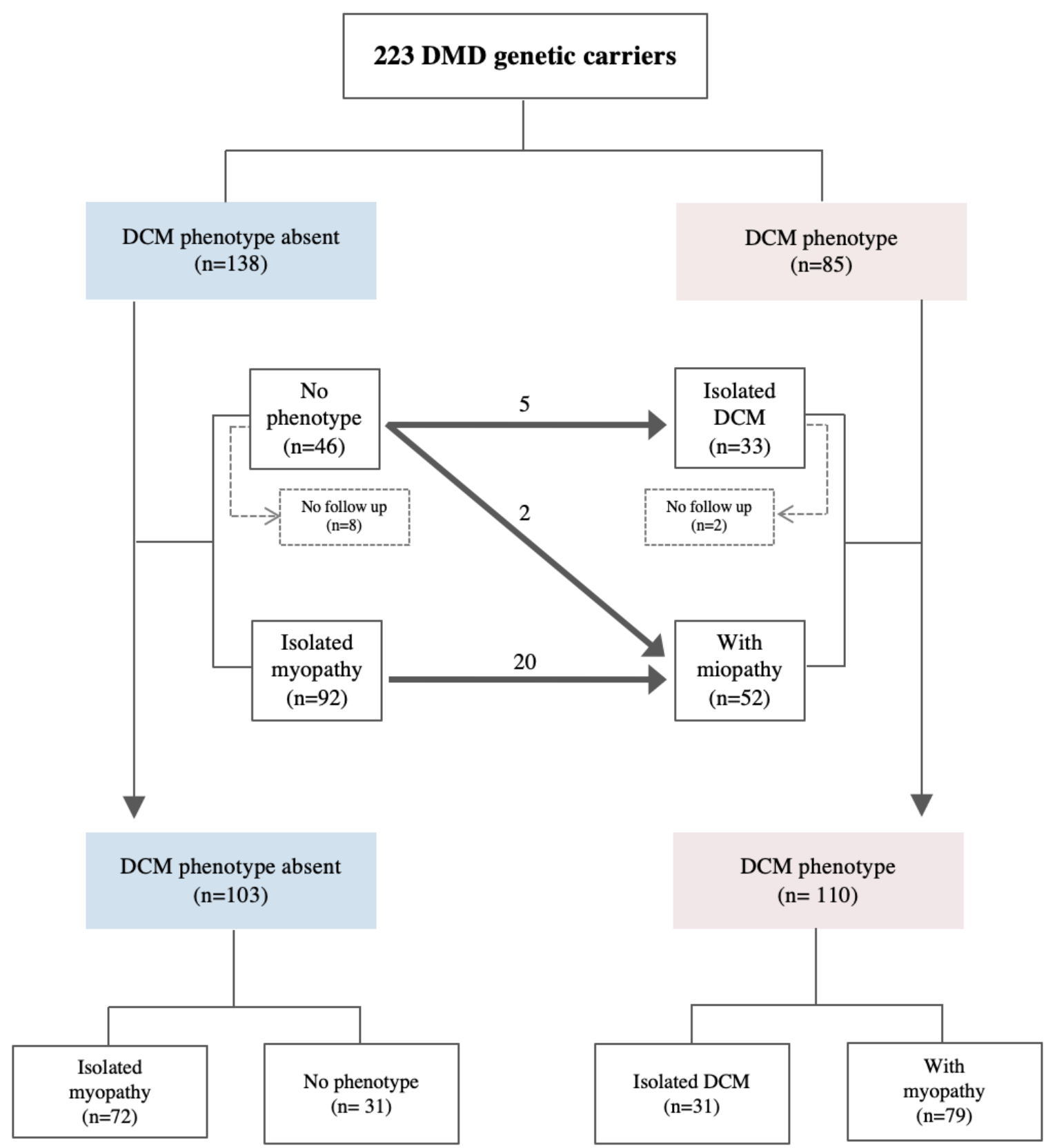


Figure 2. Survival free of MACE. A. Freedom from MACE in all patients included in the study. B. According to the presence of DCM. C. According to muscular phenotype.

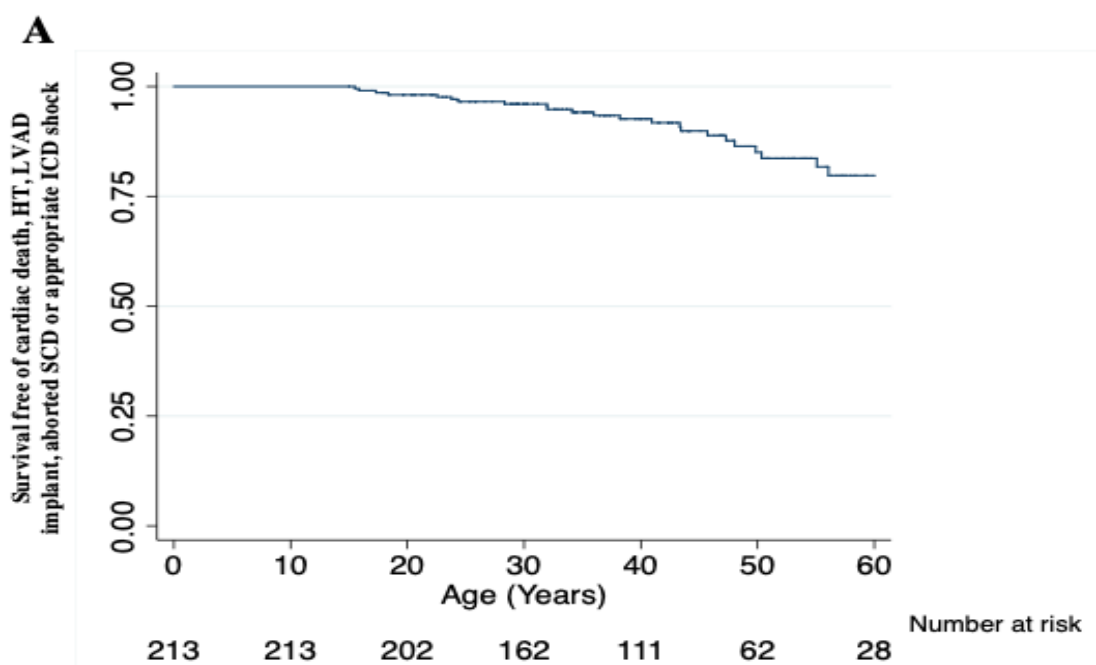

B

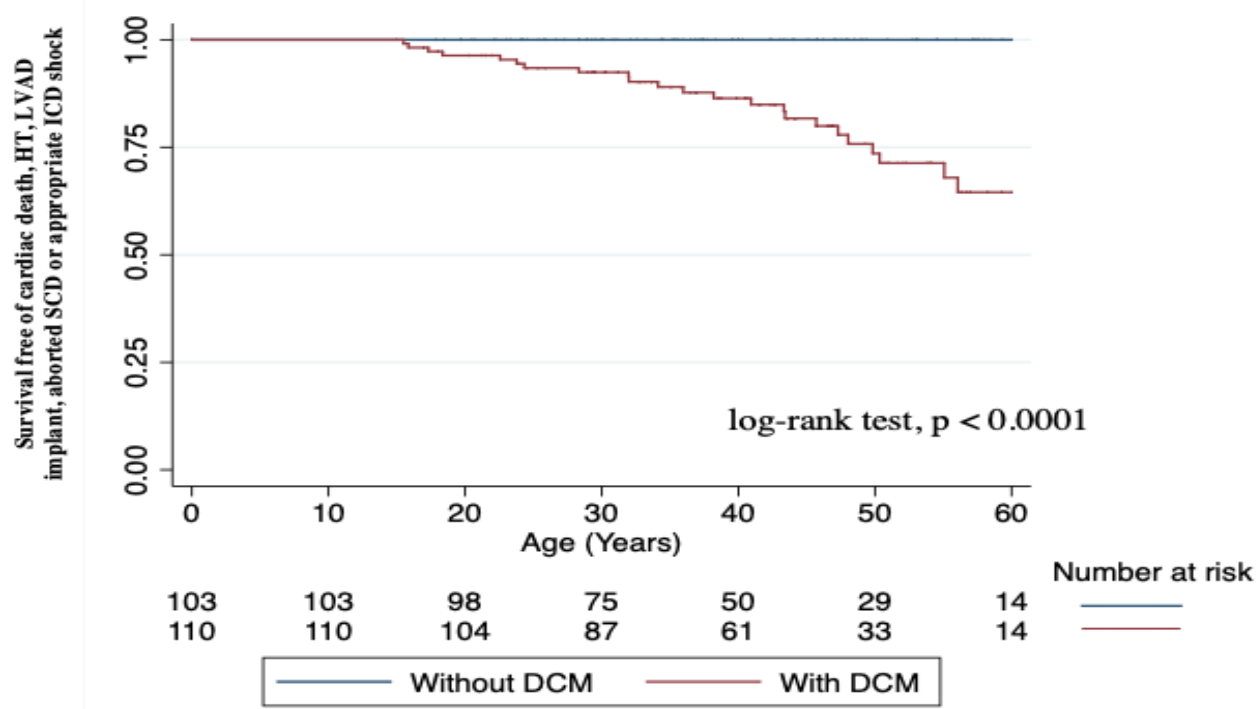

$\mathbf{C}$
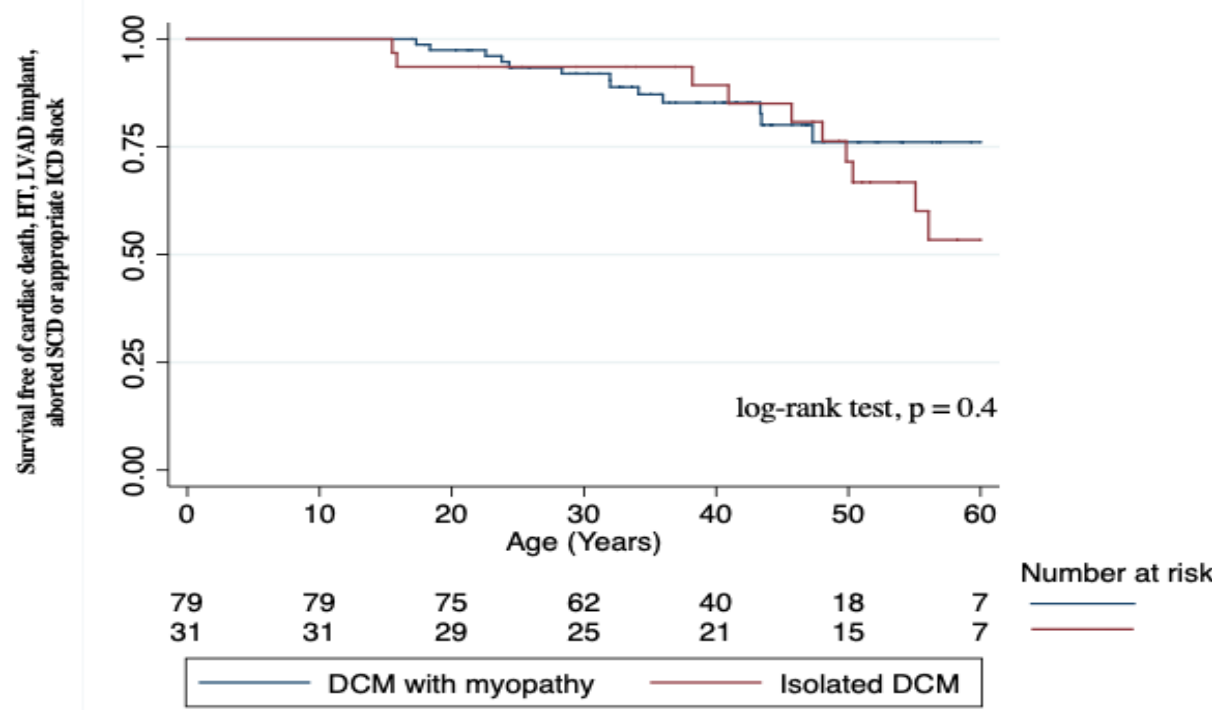
Figure 3. Age at DCM diagnosis in individuals with $D M D$ mutations according to gender. Box-plot diagram of age of DCM diagnosis reveals that DCM diagnosis in men is significantly earlier than in women $(\mathrm{p}<0.001)$.

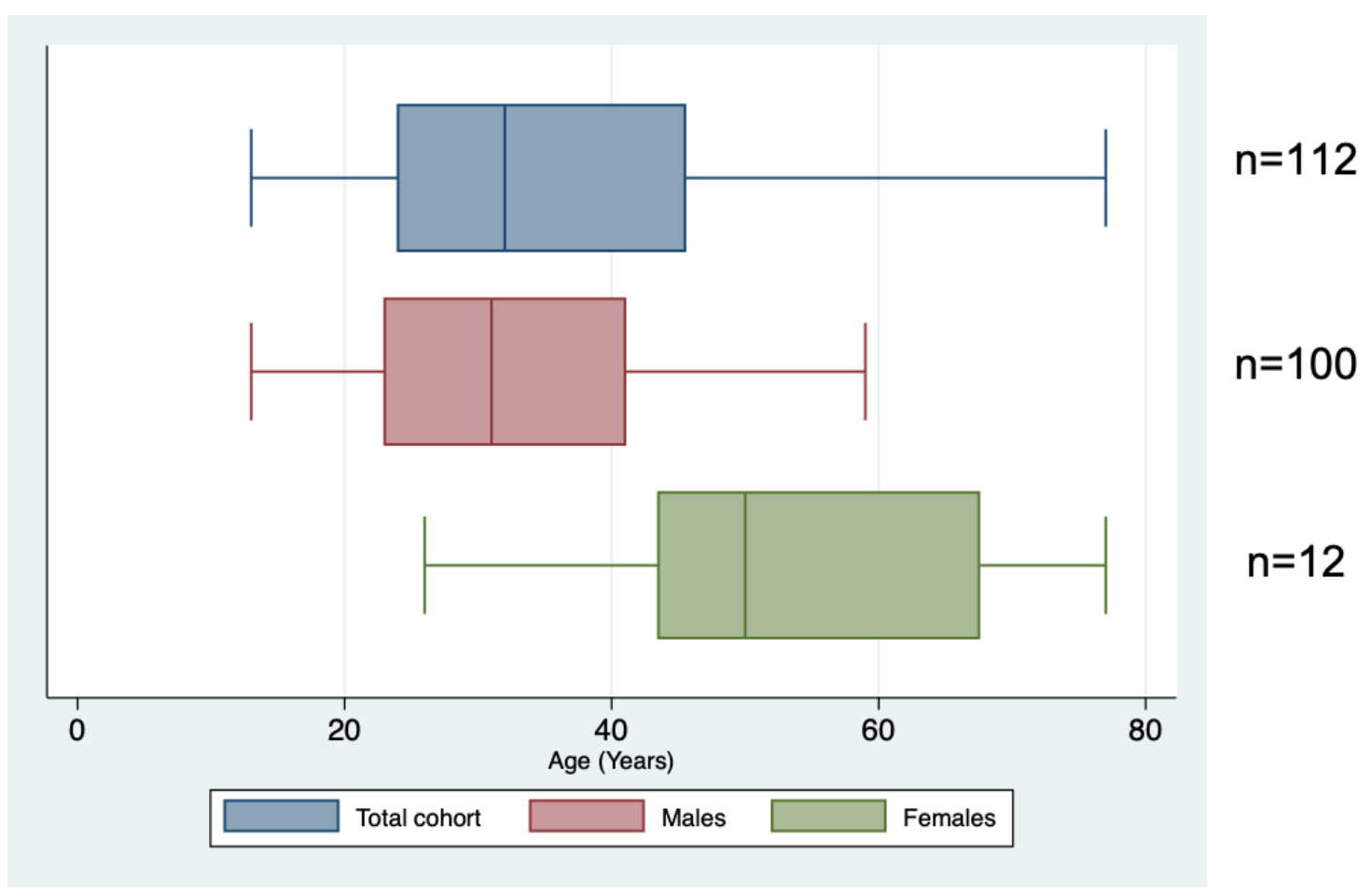


Graphical Abstract. Main Characteristics of DMD-associated DCM. $D M D$-associated DCM shows incomplete penetrance and a later diagnosis among DCM patients without concomitant skeletal myopathy. DMD mutations result in progressive cardiomyocyte death and fibrosis, leading to DCM. DCM onset is the major determinant of prognosis. Prognosis is similar among DMD DCM patients with or without skeletal muscle involvement.

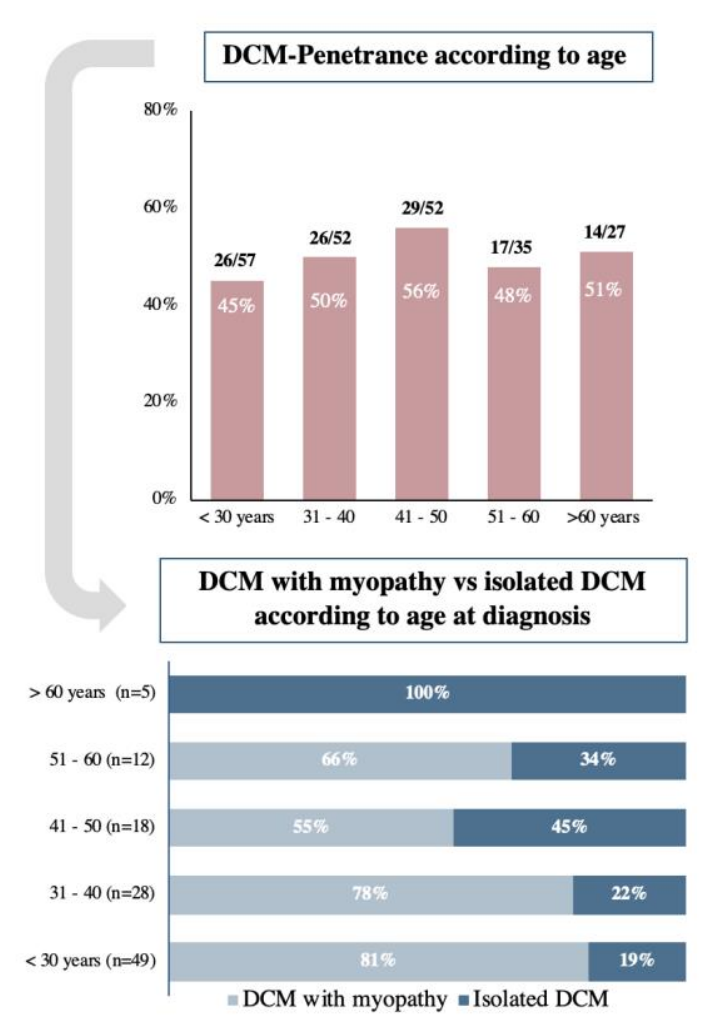

MODERATE PENETRANCE LATER DIAGNOSIS IN ISOLATED DCM
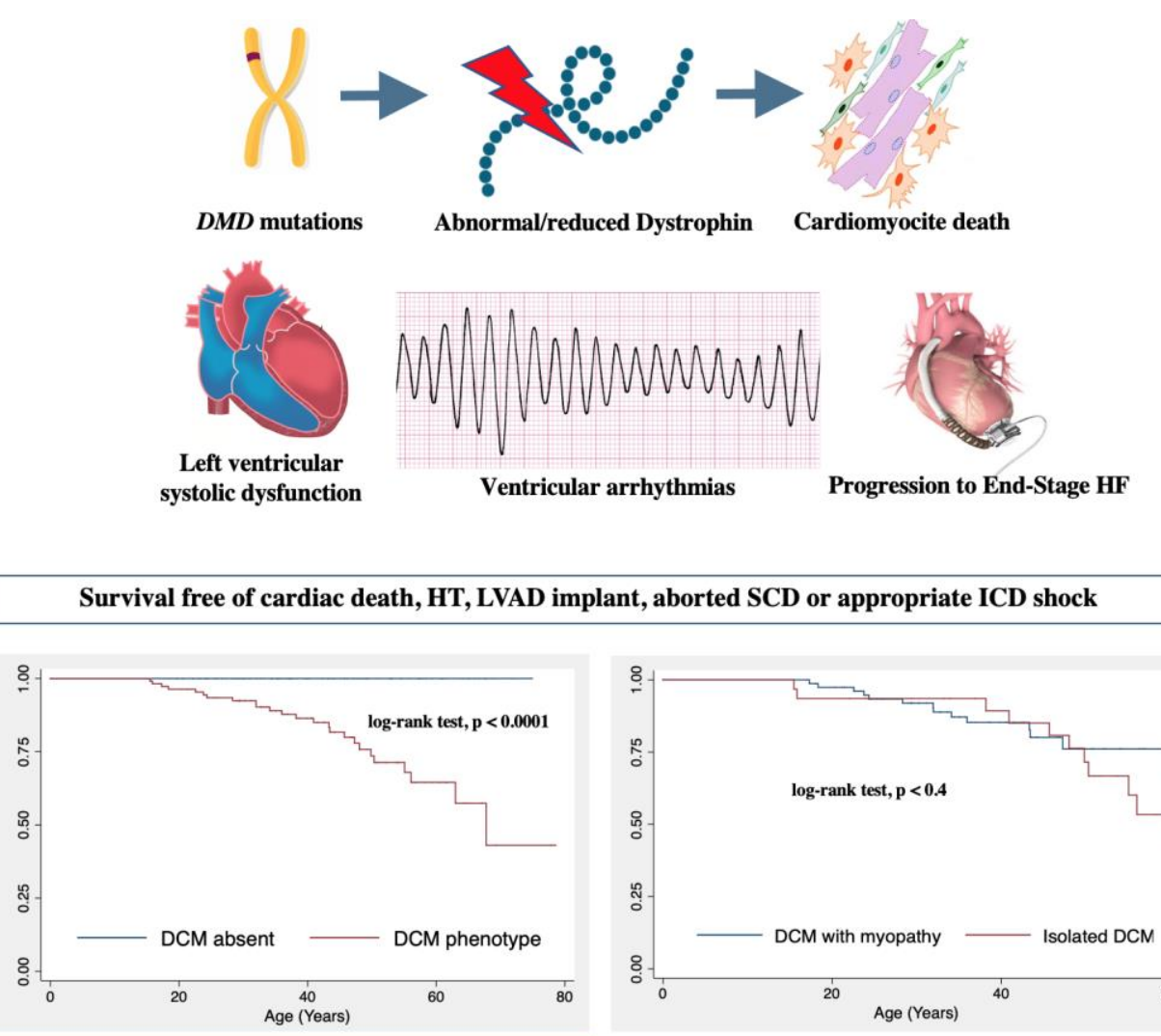

DCM PHENOTYPE IS THE MAJOR DETERMINANT OF PROGNOSIS

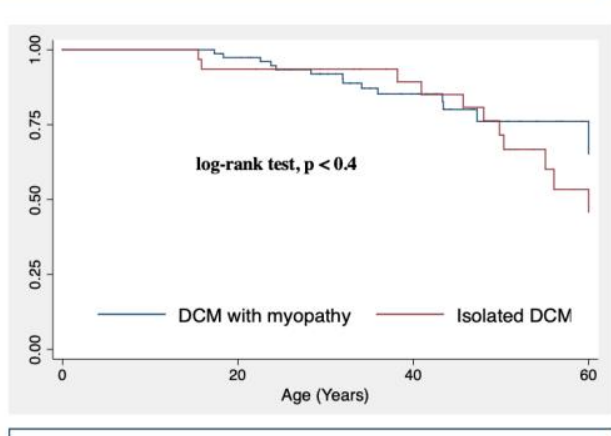

SIMILAR SURVIVAL IN DCM WITH SKELETAL MYOPATHY AND ISOLATED DCM

DCM: dilated cardiomyopathy; DMD: dystrophin gene; HF: heart failure; HT: heart transplantation; ICD: implantable cardioverter-defibrillator; LVAD: left ventricular assist device; SCD: sudden cardiac death. 